TRANSPARENCIA

Y DIGNIDAD

La comunicación

\section{financiera socialmente}

\section{responsable}

\section{Dr. Rafael López Lita}

\section{Catedrático en Comunicación Audiovisual y \\ Publicidad \\ Departamento de Ciencias de la Comunicación. Facultad de Cien- cias Humanas y Sociales. Universitat Jaume I. Avda. Sos Baynat, s/n, 12071, Castellón de la Plana (España) - Tlfns: (+34) 964729827/(+34)649030403 - Email: rlopez@com.uji.es}

\section{Dra. Magdalena Mut Camacho}

\section{Profesora}

Departamento de Ciencias de la Comunicación. Facultad de Ciencias Humanas y Sociales. Universitat Jaume I. Avda. Sos Baynat, s/n, 12071, Castellón de la Plana (España) - Tlfns: (+34)964729827/ (+34)696551862 - Email: magda.mutecom.uji.es

\section{Resumen}

La sociedad hoy en día exige a las empresas el afianzamiento de unos valores que van desde el respeto de la naturaleza, la ética y las cuestiones sociales.

Todas estas exigencias llevadas a los productos financieros plantean un panorama complejo que necesita transparencia en pos de dignidad. Los

\section{Palabras clave}

Comunicación financiera,

responsabilidad social corporativa, inversión socialmente responsable, ética,

comunicación corporativa

Key Words

Financial communication, corporate social responsibility, socially responsible investment, ethics,

corporate communications

\section{Abstract}

Today the society requires to the companies values like respect nature, ethics and social issues. All these requirements make necessary that financial products have transparency and dignity. The products should provide full information to enable the

consumer to understand without doubt the reality of its contents, and information necessary to evaluate their expectations.

This requires operators to be responsible, allowing Financial Communication occupies a basic role to play a dual task: shaping public with suffi-

cient knowledge of products and markets to discern good for them and present the products with the proper level of transparency. 
productos se presentan en formas mucho más complejas, deben proporcionar una información plena que permita al consumidor medio entender sin dudas la realidad de su contenido, y elementos necesarios para la evaluación de sus expectativas.

Para ello es necesario que los operadores sean responsables, permitiendo que la Comunicación Financiera ocupe un protagonismo básico para desempeñar una doble tarea: formar públicos con conocimientos suficientes de productos y mercados para poder discernir que les conviene y presentar los productos con el debido nivel de transparencia.

\section{Introducción}

La crisis forma parte del ciclo económico. Incluso es algo positivo, ya que elimina del mercado aquellos operadores menos competitivos y deja hueco a los más eficientes, lo que abre el camino al progreso, al cambio, a la rectificación. Pero cuando a ésta se une un factor nuevo como las conductas indecorosas y sin ética, da como resultado un orden financiero alterado de tal modo, que es imprevisible el tiempo que tardará en volver a cumplir eficientemente su verdadera función.

En esta época de turbulencias en el mundo financiero, entre estas múltiples alteraciones que darán lugar a surtidos planteamientos, se observa el inicio de la mutación de consumidores en ciudadanos, hasta llegar al ciudadano digno.

Resulta evidente que el retorno al buen funcionamiento del sistema financiero es la piedra angular sobre la que descansa el futuro del estado del bienestar. Para ello es imprescindible lograr un alto grado de transparencia que permita conocer de forma indubitable el contenido real en términos monetarios y de riesgo de cada uno de los productos financieros que aparecen en los mercados.

El grado de desarrollo de un sistema económico depende del buen funcionamiento de su sistema financiero como nos ha mostrado recientemente la realidad y, desventuradamente, ha puesto de manifiesto gran parte de las carencias manifestadas anteriormente.

El logro de la transparencia de los productos financieros ha sido una constante preocupación del grupo de investigación "Observatorio en nuevas tendencias y procesos de innovación en comunicación” del Departamento de Ciencias de la Comunicación de la Universitat Jaume I de Castellón.

Maximizar la eficiencia de los productos financieros en términos económicos $\mathrm{y}$ sociales, potenciando los procesos de creación de riqueza, que en definitiva, son el factor de dinamización de la economía y por lo tanto del progreso, resulta esencial para lograr los equilibrios necesarios y, en consecuencia, contribuir a mejorar la calidad de vida. 
La exigencia de los ahorradores impulsó un importante grado de renovación de los productos financieros, llevando a los emisores a un esfuerzo de imaginación para satisfacer una demanda cada vez más sofisticada. El problema es que en demasiadas ocasiones se ha alejado demasiado de la anteriormente expresada necesidad de transparencia.

La realidad nos lleva a percibir dicha exigencia de forma que sin prescindir de factores meramente económicos incluya la implicación de los emisores en materias relacionadas con su conducta con respecto a temas diversos que se aglutinan alrededor de un eje vertebrador de carácter ético.

El fin último de dichas inversiones debe ser respetuoso de los nuevos valores que comienzan a perfilar las sociedades avanzadas del mundo occidental.

Valores que van desde el respeto de la naturaleza, la forma de abordar los conflictos, las relaciones con sus públicos, etc. hasta un compromiso permanente con su entorno.

Pero, sobre todo debemos plantearnos un elevado nivel de exigencia, de modo que al mismo tiempo que los productos financieros se presentan en formas mucho más complejas, deben proporcionar una información plena que permita al consumidor medio entender sin ningún género de dudas la realidad de su contenido y elementos necesarios para la evaluación de sus expectativas.

Para ello es necesario que los operadores sean socialmente responsables, término que a poco que meditemos intuiremos que tiene un alcance mucho más profundo que la mera responsabilidad social corporativa ${ }^{i}$.

Evidentemente, poco o nada de lo anterior ha sido tenido en consideración por determinados agentes del mercado lo que ha provocado, como no podía ser de otra manera, la situación de deterioro en la que nos encontramos.

\section{Objetivos}

Posiblemente nos debemos decantar por una profundización de las ideas anteriores, permitiendo que la Comunicación Financiera ocupe un protagonismo básico para desempeñar una doble tarea:

- Formar públicos con conocimientos suficientes de productos y mercados pa- ra poder discernir que les conviene en cada momento en función de su perfil de inversor fundamentalmente en función de riesgo y liquidez.

- Presentar los productos con el debido nivel de transparencia. 


\section{Metodología}

En 2005 se inició el proyecto de investigación "La comunicación de nuevos productos financieros socialmente responsables: propuestas para su desarrollo y aplicación España”, durante varios años el equipo investigador ha ido trabajando con unos claros objetivos y con una metodología de trabajo común que ha dado como resultado diferentes enfoques y resultados, como el que se presenta en este artículo.

Las conclusiones que se exponen en el siguiente escrito manan del estudio sobre el tratamiento comunicativo que reciben los productos financieros socialmente responsables en la actualidad. En una primera fase, una recopilación de materiales periodísticos y publicitarios sirvió para elaborar el material documental del trabajo de campo en su primera fase. En la siguiente fase, con la panorámica sobre el estado de la cuestión, se indagó cada producto financiero para tener la referencia actual sobre la comunicación y la repercusión mediática que este tipo de productos está obteniendo en los medios. En su fase final, la investigación derivó en la planificada reflexión junto con expertos seleccionados para la puesta en común de conclusiones sobre la situación actual y sus propuestas de desarrollo.

\section{La ética legitima}

En un principio, cuando se extendió la sospecha sobre la ética y legalidad de las prácticas financieras y de negocio de la comunidad empresarial, con escándalos como la burbuja de Internet o los relacionados con el mal comportamiento de los consejeros y directivos de empresas como Enron/Andersen, Ahold, Tyco o Vivendi, se desplomó la confianza de la opinión pública en las grandes corporaciones privadas. Principios de los 2000 fue una época de griterío sobre especulación, abuso de poder, discriminación salarial, contaminación, escándalos protagonizados por los altos directivos, y todas eran empresas que se consideraban sólidas y de confianza. Esto propició el ambiente extremadamente crítico de toda la opinión pública $\mathrm{y}$, desde el otro lado, desembocó en una empresa que percibió la importancia de la reputación y su valor como activo intangible.

Es posible que la nueva configuración empresarial en la que desembocó todo esto sea un ciclo más de la macroeconomía, quizá sea jactancioso achacarle como único motivo del cambio, la reacción contra el cinismo y la inmoralidad de los directivos. Pero fue la gota que colmó el vaso, a partir de ese momento, se empezó a ver el verdadero problema: la falta de confianza en las 
empresas sospechosas de su ética y de su moralidad.

El advenimiento fue imparable, la opinión pública, que siempre se ha cuestionado la racionalidad empresarial y, por extensión, la función social o la falta de función social de los negocios, pasó a manifestar de forma activa, sonora y visible sus dudas. De la combinación perversa de crisis moral de las empresas y de crisis de confianza de la sociedad, la reflexión hacia la responsabilidad corporativa estaba abierta por muchos frentes.

A partir de ahí, la empresa integró la buena administración de las cosas: buena reputación, ética corporativa, legitimidad social y conciencia medioambiental. Este era el camino, mantenerse en la fórmula que el Catedrático López Lita llama "doble liderazgo", esto es ser líder en costes al tener el mejor producto/servicio al mejor precio y amparado además con una imagen potente, abrigada por una marca conocida y ahora también admirada. Conseguir una empresa que despierta mayor confianza y disfruta de una mayor legitimidad social, se consigue desde su producto/servicio y desde su capacidad de cumplir las expectativas de sus accionistas, sus clientes, los gobiernos, los mercados de capitales y la ciudadanía.

Desde ese momento, la empresa integró en su estrategia de negocio nuevas responsabilidades, más allá de las obligaciones legales y fiscales. La comunicación de esta nueva cultura corporativa que surgía con fuerza en el seno de la organización fue pasando de informativa a reveladora.

En España hay alta conciencia por parte de las empresas, sobre todo cotizadas, con respecto al comportamiento responsable. El índice FTSE4Good IBEX tiene criterios de inclusión de la responsabilidad de las empresas, éstas han de ser completamente transparentes y accesibles al público. El índice puede ser utilizado:

- Para permitir a los inversores responsables identificar e invertir en compañías que cumplen con los estándares reconocidos a nivel mundial de responsabilidad corporativa

- Para proporcionar a los administradores de activos, con una inversión responsable de referencia, una herramienta para el desarrollo de productos de inversión responsable.

- Como una solución para los administradores de fondos públicos de pensiones, que en el futuro, es probable que sean requeridos por la legislación española para invertir el 10\% de los fondos de pensiones públicos en inversión responsable.

- Para permitir a los inversores capitalizar los beneficios de la responsabilidad de las empresas buenas (por ejemplo, ecoefficiencies, mejorar la imagen de marca, etc.)

- Como una herramienta de participación para alentar a las empresas a ser más responsables. 
La práctica de la Responsabilidad Corporativa también se ha puesto en el punto de mira del sector financiero, no únicamente como actitud, sino como producto. Un producto financiero socialmente responsable es un activo financiero que, aparte de tratar de ofrecer la máxima rentabilidad y liquidez con el mínimo riesgo, se les exige además unos valores morales y éticos. Se trata de una filosofía de inversión que mezcla objetivos éticos, medioambientales y sociales con objetivos financieros y en el que además confluyen cuatro factores fundamentales: rentabilidad, liquidez, seguridad y dignidad ${ }^{\text {iii. }}$

En este sentido, los productos financieros socialmente responsables comportan un plus de transparencia. Cualidad que se dará en tanto en cuanto se utilice la comunicación financiera de forma eficiente. Esta actitud de transparencia también se puede considerar como ética al servicio del producto ético. Es una doble legitimación.

\section{El mercado de productos financieros socialmente responsables}

Las circunstancias actuales resaltan la necesidad de considerar con seriedad un nuevo factor de los productos financieros que no puede ni debe pasar desapercibido para todos aquellos que tengan a su cargo responsabilidades directivas en entidades financieras y en general en empresas o instituciones que recurran a los mercados financieros en búsqueda de financiación e incluso en lideres de opinión: periodistas, analistas, consultores, etc. conscientes de los importantes cambios a los que los acontecimientos acaecidos con posterioridad a agosto del 2007 nos deben llevar.

Los operadores han acudido tradicionalmente a los mercados organizados en búsqueda de recursos financieros desarrollando una estrategia de diversificación encaminada a lograr que su oferta pueda ajustarse a la curva de demanda de dichos productos en función de tres variables fundamentales: rentabilidad, liquidez y solvencia. En función de los mismos los diversos tipos de consumidor pretendían maximizar su utilidad priorizando unos sobre otros de forma que el producto se ajuste mejor a sus necesidades y a su perfil respecto al riesgo.

En la nueva etapa que se abre no deberán descuidar la emergente cuarta variable: la cualidad de producto socialmente responsable, lo que implica algo más allá de la responsabilidad social que resumiendo en una sola palabra la podríamos sintetizar como dignidad.

Es cierto que podría pensarse que estamos hablando de una de las características de la ya tradicional solvencia, variable conside- 
rada con anterioridad, pero la solvencia se queda en una simple probabilidad de recobrar la inversión y sus rendimientos, mientras que la dignidad recoge la probabilidad de que el uso de la inversión resulte adecuada dentro de un marco ético preestablecido y por supuesto una garantía de transparencia.

En el ámbito de productos emitidos por Administraciones Públicas, podría llegarse fácilmente a la reformulación de producto socialmente responsable en términos similares, es decir, en función de la calidad de gobierno - gobernanza - respetuoso con los valores antes expresados, pero sobre todo que representen una calidad democrática global, es decir, no sólo con respecto a sus propios ciudadanos.

Las empresas además de lanzar sus propios productos o servicios al mercado (los que constituyen el objeto de su actividad, que en la mayor parte de los casos serán no financieros), puede tratar de colocar en los mercados financieros productos para optimizar su propia financiación.

Cualquier empresa que quiera garantizar su supervivencia en el mercado no descuidará la atención a sus antiguos clientes, con el doble objetivo de continuar contando con ellos como clientes potenciales y de mantener el prestigio y la calidad del producto o servicio frente al mercado.

Idéntica conducta deberá llevar con sus productos financieros, ya que su historia posterior a su colocación en un mercado primario, es decir, su conducta en los mercados secundarios, que en principio parece no afectar, resultará determinante para futuras colocaciones.

No obstante, resulta obligado complementar algunos de los conceptos vertidos, ya que la aparición de operadores en los mercados, para mediante la colocación de sus productos obtener financiación, no queda reservada, ni mucho menos, para el ámbito de las empresas.

Como es bien sabido, el Estado, directamente o a través de sus organismos de distinta naturaleza, concurre a dicho mercado para colocar sus empréstitos, que también aparecen bajo diversas formas $y$ presentaciones, con el mismo objetivo de las empresas: adaptarse todo lo posible a la curva de demanda de cada momento.

De algún modo, también el Estado debe cuidar la evolución y comportamiento de sus emisiones pasadas en los mercados secundarios, siguiendo similares razonamientos antes empleados.

En resumen, es imprescindible que los oferentes de productos financieros incluyan en su perfil cargas importantes de transparencia ya que con mayor frecuencia los inversores tendrán en mayor aprecio y consideración el cuarto factor ético: la dignidad del emisor, transmitida al producto.

De hecho la marca financiera cobra una inusitada importancia a la luz de los últimos acontecimientos, siempre que se encuentre 
respaldada por una trayectoria de dignidad.

\section{La demanda de productos financieros socialmente responsables}

El comprador de productos financieros adquiere cada vez mayor conciencia de su propia identidad y comienza a sospechar en la fuerza que emana de su condición de ciudadano, lo que le va a permitir ejercer derechos básicos, como el de la información, en un ámbito tradicionalmente descuidado.

En este sentido, y tutelando al ciudadano, desde el gobierno se han dado ejemplos de avance, como la creación en 2008 del Consejo Estatal de Responsabilidad Social de la Empresa. Se trata de un órgano consultivo con un relevante papel en la orientación al gobierno en su apuesta por la Responsabilidad Social, entre sus tareas se haya el implicarse en la inversión socialmente responsable.

La comunicación financiera es una herramienta imprescindible para reforzar $y$ afianzar la conciencia ciudadana, que le permite ejercer su derecho de ver respeta- da su propia identidad como ciudadano responsable e interesado en el bien común.

Los acontecimientos acaecidos en los mercados últimamente han acelerado previsiblemente la mutación de consumidores en ciudadanos dignos, y deberán reforzar su consideración en la descrita variable "dignidad” en su proceso de toma de decisiones.

La comunicación financiera actúa como catalizador indispensable del proceso.

De lo anterior parecen desprenderse dos consecuencias:

- Se llamen como se llamen, los productos financieros del futuro deberán ser socialmente responsables si quieren merecer el aprecio de los demandantes.

- La comunicación financiera es el instrumento necesario para armar la estructura intelectual sobre la que se soportará el nuevo concepto.

\section{Conclusiones: El futuro proyecta nuevos paradigmas}

Dada la evolución de los acontecimientos y en función de la situación actual e incluso previsible para los próximos años, pode- mos apreciar dos nuevos paradigmas relacionados con los productos financieros socialmente responsables: 
- Los relacionados con inspirar confianza ante los numerosos casos de información financiera inexacta cuando no fraudulenta.

- Los derivados de introducir en su metodología los nuevos valores derivados de la creciente importancia de productos financieros responsables procedentes de emisores socialmente responsables, es decir el descrito cuarto factor: dignidad.

Contando con los anteriores conceptos, la nueva comunicación financiera especializada en los productos socialmente responsables deberá tener ineludiblemente las siguientes características:

- Presentación al mercado de los diversos productos financieros emitidos por empresas y entidades, incluso el Estado, contribuyendo al diseño transparente y responsable de los mismos.

- Colocación en dichos mercados de tales productos, de acuerdo con las condiciones de emisión, tomando en consideración los nuevos paradigmas.

- Mantenimiento, durante toda la vida de los productos, de su imagen digna y positiva, para la que resulta esencial que los emisores lleven a cabo una adecuada gestión de su reputación corporativa comprometida con valores éticos.

Deberán encontrarse presentes en todas las acciones de comunicación, cualquiera que sean las herramientas empleadas, dirigidas y encaminadas, tanto desde entidades y empresas del sector público como del privado y utilizará cualquier herramienta de comunicación que pueda resultar idónea, sin que su propia especificidad le suponga de antemano limitación de ninguna naturaleza adaptando, y dotando de personalidad propia algunas de las técnicas de comunicación generalmente empleadas, creando figuras como la de la oficina de atención al accionista y otras similares que no tendrían sentido fuera del ámbito descrito.

Como al principio se mencionaba, no puede pensarse en una sociedad estable y con un razonable nivel de desarrollo económico y social sin que sus mercados básicos, entre ellos el financiero, funcionen en forma socialmente eficiente, para ello no se pueden perder de vista los cuatro factores descritos: rentabilidad, liquidez, seguridad y "dignidad".

La comunicación financiera actúa en todo momento como la fuerza que activa y dinamiza cualquier mercado, y en el caso que nos ocupa no constituye una excepción, es una de las fuerzas confluyentes, necesaria pero no suficiente, para que los mercados financieros alcancen la flexibilidad y transparencia necesaria, para la optimización de la función de financiación.

Precisamente tenemos muy a la vista, en los momentos que vivimos, la incidencia que el funcionamiento imperfecto del mercado financiero puede tener en el grado de inflación estructural del sistema económico y muy directamente en la generación de riqueza, y en consecuencia en los sacrificios 
que serán necesarios para lograr reequilibrar tal efecto nocivo.

La comunicación financiera puede contribuir a reducir tales inconvenientes, ayudando a la existencia de mercados más competitivos con una clara ventaja para todos, en los que los productos financieros socialmente responsables constituyan el eje vertebrador del equilibrio.
Su misión estratégica fundamental deberá consistir en alertar a los consumidores y provocar su exigencia, como ciudadanos, para que en ningún momento ni emisores ni reguladores dejen de tener presente la ineludible necesidad de presentar a los mercados exclusivamente productos financieros socialmente responsables.

\section{Referencias}

BARREDA, J.C. y otros (2008): El papel de la comunicación especializada en el desarrollo de los productos financieros socialmente responsables en España en López Lita, Rafael (2008): La comunicación de nuevos productos financieros socialmente responsables. Madrid, Ciencias Sociales.

BENAVIDES, J. y otros (Eds.) (2008): Nuevas tendencias de la Comunicación. Madrid, colección Libros Complutenses, Serie Comunicación.

BERNAD, E. y BOLDÓ, M.C. (2008): Reflexiones entorno al tratamiento jurídico de los productos financieros socialmente responsables en los casos de publicidad engañosa en López Lita, Rafael (2008): La comunicación de nuevos productos financieros socialmente responsables. Madrid, Ciencias Sociales.

\section{COMISIÓN DE LAS COMUNIDADES}

EUROPEAS (2001): Libro Verde. Fomentar un marco europeo para la responsabilidad social de las empresas. Bruselas, COM, 366.

CORTINA, A. (2003): Por una ética del consumo. Madrid, Taurus.

ECONOMISTAS SIN FRONTERAS (2007):

Cómo fomentar la inversión socialmente responsable en España. Madrid, UNED.

\author{
EUROPEAN SOCIAL INVESTMENT FORUM \\ (2006): European SRI Study, recuperado de \\ http: / / www. eurosif.org/publications/sri studies \\ FERNANDEZ OLIT, B. y otros (2008): Cómo \\ fomentar la inversión socialmente responsable en España. \\ Madrid, Colección Varia, UNED, Economistas sin \\ Fronteras y Ministerio de Trabajo y Asuntos \\ Sociales.
}

LÓPEZ LITA, Rafael (2000): Comunicación, clave del bienestar social. Madrid, Drac

PIZZOLANTE, I. (2009): De la responsabilidad social empresarial a la empresa socialmente responsable. Madrid, Ciencias Sociales.

\section{Cita de este artículo}

LÓPEZ LITA, R.L. y MUT CAMACHO, M. (2010) Transparencia y dignidad. La comunicación financiera socialmente responsable. Revista Icono14 [en línea] 1 de Julio de 2010, Año 8, Vol. 2. pp. 153-163. Recuperado (Fecha de acceso), de http://www.icono14.net

\section{NOTAS}

${ }^{\text {i }}$ Pizzolante, I. (2009): De la responsabilidad social empresarial a la empresa socialmente responsable. Madrid, Ciencias Sociales. 
${ }^{i i}$ López Lita, Rafael (2000): Comunicación, clave del bienestar social. Madrid, Drac, pp. 121-123.

iii Bernad, E. y Boldó, M.C. (2008): Reflexiones entorno al tratamiento jurídico de los productos financieros socialmente responsables en los casos de publicidad engañosa en López Lita, Rafael (2008): La comunicación de nuevos productos financieros socialmente responsables. Madrid, Ciencias Sociales. 